\title{
Distinct Compartmentalization of Dentin Matrix Protein 1 Fragments in Mineralized Tissues and Cells
}

\author{
Izabela Maciejewska ${ }^{a}$ Disheng Qina Bingzhen Huang ${ }^{a}$ Yao Sun ${ }^{a}$ \\ Gabrielle Mues $^{\mathrm{a}}$ Kathy Svoboda ${ }^{\mathrm{a}}$ Lynda Bonewald $^{\mathrm{b}}$ William T. Butler ${ }^{\mathrm{a}}$ \\ Jerry Q. Feng ${ }^{a} \quad$ Chunlin Qin ${ }^{a}$ \\ ${ }^{a}$ Department of Biomedical Science, Baylor College of Dentistry, Texas A\&M University System Health Science \\ Center, Dallas, Tex., and ' ${ }^{\mathrm{b}}$ School of Dentistry, University of Missouri at Kansas City, Kansas City, Mo., USA
}

\section{Key Words}

Dentin matrix protein $1 \cdot$ Proteolytic processing •

Localization $\cdot$ Mineralized tissues $\cdot$ Osteocytes

\begin{abstract}
Dentin matrix protein 1 (DMP1) has been shown to be critical for the formation of dentin and bone. However, the precise pathway by which DMP1 participates in dentinogenesis and osteogenesis remains to be clarified. DMP1 is present in the extracellular matrix of dentin and bone as processed $\mathrm{NH}_{2}-$ and $\mathrm{COOH}$-terminal fragments. The $\mathrm{NH}_{2}$-terminal fragment occurs as a proteoglycan, whereas the $\mathrm{COOH}$-terminal fragment is highly phosphorylated. The differences in biochemical properties suggest that these fragments may have different tissue and cell distribution in association with distinct functions. In this study, we analyzed the distribution of the $\mathrm{NH}_{2}$ - and $\mathrm{COOH}$-terminal fragments of DMP1 in tooth, bone, osteocytes as well as MC3T3-E1 and HEK-293 cells. Immunohistochemical analyses were performed using antibodies specific to the $\mathrm{NH}_{2}$ - or $\mathrm{COOH}$-terminal region of DMP1. Clear differences in the distribution of these fragments were observed. In the teeth and bone, the $\mathrm{NH}_{2}$-terminal fragment was primarily located in the nonmineralized predentin and
\end{abstract}

cartilage of the growth plate, while the $\mathrm{COOH}$-terminal fragment accumulated in the mineralized zones. In osteocytes, the $\mathrm{NH}_{2}$-terminal fragment appeared more abundant along cell membrane and processes of osteocytes, while the $\mathrm{COOH}$-terminal fragment was often found in the nuclei. This pattern of distribution in cellular compartments was further confirmed by analyses on MC3T3-E1 and HEK-293 cells transfected with a construct containing DMP1 cDNA. In these cell lines, the $\mathrm{COOH}$-terminal fragment accumulated in cell nuclei, while the $\mathrm{NH}_{2}$-terminal fragment was in the cytosol. The different distribution of DMP1 fragments indicates that these DMP1 variants must perform distinct functions.

Copyright $\odot 2008$ S. Karger AG, Basel

\begin{tabular}{ll}
\hline Abbreviations used in this paper \\
\hline DMP1 & dentin matrix protein 1 \\
DMP1-PG & proteoglycan form of DMP1 \\
HEK-293 & human embryonic kidney 293 cell line \\
IHC & immunohistochemical \\
MC3T3-E1 & mouse preosteoblastic cell line
\end{tabular}

\section{KARGER}

() 2008 S. Karger AG, Basel

Fax +4161306 1234

E-Mail karger@karger.ch

www.karger.com
Accessible online at: www.karger.com/cto
Dr. Chunlin Qin

Department of Biomedical Sciences, Baylor College of Dentistry

Texas A\&M University System Health Science Center

3302 Gaston Avenue, Dallas, TX 75246 (USA)

Tel. +1 214828 8292, Fax +1 214874 4538, E-Mail cqin@bcd.tamhsc.edu 


\section{Introduction}

Dentin matrix protein 1 (DMP1), originally postulated to be dentin specific [George et al., 1993], was later detected in bone [Hirst et al., 1997; MacDougall et al., 1998]. The distinctive feature of DMP1 is the presence of many acidic domains, a property that implicates it is a possible participant in regulating matrix mineralization. The importance of DMP1 in biomineralization has been demonstrated by a number of studies [Narayanan et al., 2001; Ye et al., 2004, 2005; Feng et al., 2006].

Although the isolation of the full-length form of DMP1 has been unsuccessful, the $\mathrm{NH}_{2}$-terminal fragment (37 $\mathrm{kDa}$ ), the proteoglycan form of the $\mathrm{NH}_{2}$-terminal fragment (DMP1-PG) and the COOH-terminal fragment (57 $\mathrm{kDa}$ ) of DMP1 have been extracted and characterized from bone and dentin [Qin et al., 2003, 2006]. These experiments demonstrated that after synthesis, DMP1 undergoes posttranslational proteolytic processing, resulting in the formation of individual fragments originating from the $\mathrm{NH}_{2}$ - and $\mathrm{COOH}$-terminal regions of the DMP1 amino acid sequence. The relative abundance of these fragments along with the lack of significant amounts of full-length DMP1 suggests that the $\mathrm{NH}_{2}$ - and $\mathrm{COOH}$ terminal fragments may be the functionally active forms of DMP1 that directly participate in biomineralization. The COOH-terminal fragment has been found to promote hydroxyapatite nucleation [Tartaix et al., 2004; He et al., 2005; Gajjeraman et al., 2007]. Additionally, it has been reported that in addition to its direct role in the formation and/or growth of hydroxyapatite crystals, DMP1, acting as a transcription factor, may be involved in regulating other genes associated with dentinogenesis and osteogenesis [Narayanan et al., 2003].

Based on the distinct biochemical properties of DMP1 fragments, we postulate that these fragments play different roles in dentinogenesis and osteogenesis. In the present study, we analyzed the distribution of the DMP1 fragments in tooth, bone and cellular compartments of osteocytes and 2 cell lines (MC3T3-E1, HEK-293). Our results showed clear differences in the distribution of the $\mathrm{NH}_{2}-$ and $\mathrm{COOH}$-terminal fragments, suggesting potent multiple roles of DMP1 in dentinogenesis and osteogenesis.

\section{Materials and Methods}

\section{Antibodies}

Initially, 2 antibodies raised against 2 synthetic peptides, GLGPEEGQWGGPSKLDSDEDS 101-121, termed anti-DMP1784 , to detect the amino-terminal fragment, and AYNHKPIG-
DQDDNDC 471-485, termed anti-DMP1-785, to detect the carboxy-terminal fragment of DMP1, were used for immunohistochemical (IHC) staining. New antibodies were generated based on the same sequences (Sigma Genosys, The Woodlands, Tex., USA) for this investigation. The newly generated antibodies were designated anti-DMP1-N-859 and anti-DMP1-C-857, respectively. The specificity of these antibodies was confirmed by Western immunoblotting using DMP1 fragments isolated from rat bone. The anti-DMP1-N-859 polyclonal antibody was used to identify the $\mathrm{NH}_{2}$-terminal fragment of DMP1 by IHC staining. A monoclonal antibody [Baba et al., 2004], generated using DMP1 COOHterminal fragment isolated from rat bone as the antigen, was designated as anti-DMP1-C-8G10.3. The anti-DMP1-C-8G10.3 and anti-DMP1-C-857 antibodies were used for identifying the $\mathrm{COOH}$-terminal fragment of DMP1 in IHC analysis.

\section{Tissue Preparation for Immunohistochemistry}

Sprague Dawley newborn, 3- and 8-week-old rats (Harlan, Indianapolis, Ind., USA) were perfused from the ascending aorta with $4 \%$ paraformaldehyde in $0.1 \mathrm{M}$ phosphate buffer. The entire head and humerus were dissected and further fixed in the same fixative for 2 days at $4^{\circ} \mathrm{C}$, followed by decalcification in $8 \%$ EDTA ( $\mathrm{pH} 7.4$ ) at $4^{\circ} \mathrm{C}$ for $3-5$ weeks. Tissues were processed for paraffin embedding and serial $8-\mu \mathrm{m}$ sections were prepared for immunohistochemistry. The animal protocol was approved by the Animal Welfare Committee of the Baylor College of Dentistry of the Texas A \& M University System Health Science Center.

\section{Immunohistochemistry Staining}

For IHC staining, all reagents were from Invitrogen (Carlsbad, Calif., USA) unless otherwise stated. Paraffin sections were pretreated with hyaluronidase solution $(1 \mathrm{mg} / \mathrm{ml})$ for $1 \mathrm{~h}$ at $37^{\circ} \mathrm{C}$, washed with PBS and incubated for $1 \mathrm{~h}$ with standard blocking solution. Primary antibodies were diluted at 1:200 in the blocking solution and applied for $1 \mathrm{~h}$ at room temperature. Alexa 488-labeled anti-rabbit and Alexa 546-labeled anti-mouse $\mathrm{F}(\mathrm{ab})_{2}$ fragments of goat IgG (secondary antibodies) were used at a dilution of 1:600 and incubated for $1 \mathrm{~h}$ at room temperature. Finally, sections were treated with TO-PRO-3 at a dilution of 1:500 for $5 \mathrm{~min}$ to stain the nuclei. The sections were coverslipped with SlowFade and assessed with a Leica SP2 scanning laser confocal microscope (Leica, Wetzlar, Germany).

\section{Cell Culture and Generation of DMP1 Construct}

A human embryonic kidney cell line 293 (HEK-293) and a murine preosteoblastic cell line (MC3T3-E1) were cultured with DMEM and $\alpha$-MEM (Invitrogen), respectively. For generation of a mammalian expression construct, the full-length mouse DMP1 cDNA [Lu et al., 2007] was first released from a pBC-KS+ construct by EcoRI digestion. The DMP1 cDNA was purified by agarose gel electrophoresis using a Quick GEL extract kit (Qiagen) and then subcloned into a pcDNA3.1 vector. This pcDNA3.1 construct was used to transfect HEK-293 or MC3T3-E1 cells. Transient transfection with the pcDNA3.1 construct was performed by using Lipofectamine 2000. Nontransfected and transfected cells were fixed with $4 \%$ paraformaldehyde for 15 min on ice. After fixation and washing with PBS, cells were stained by immunohistochemistry following the protocol described above. 

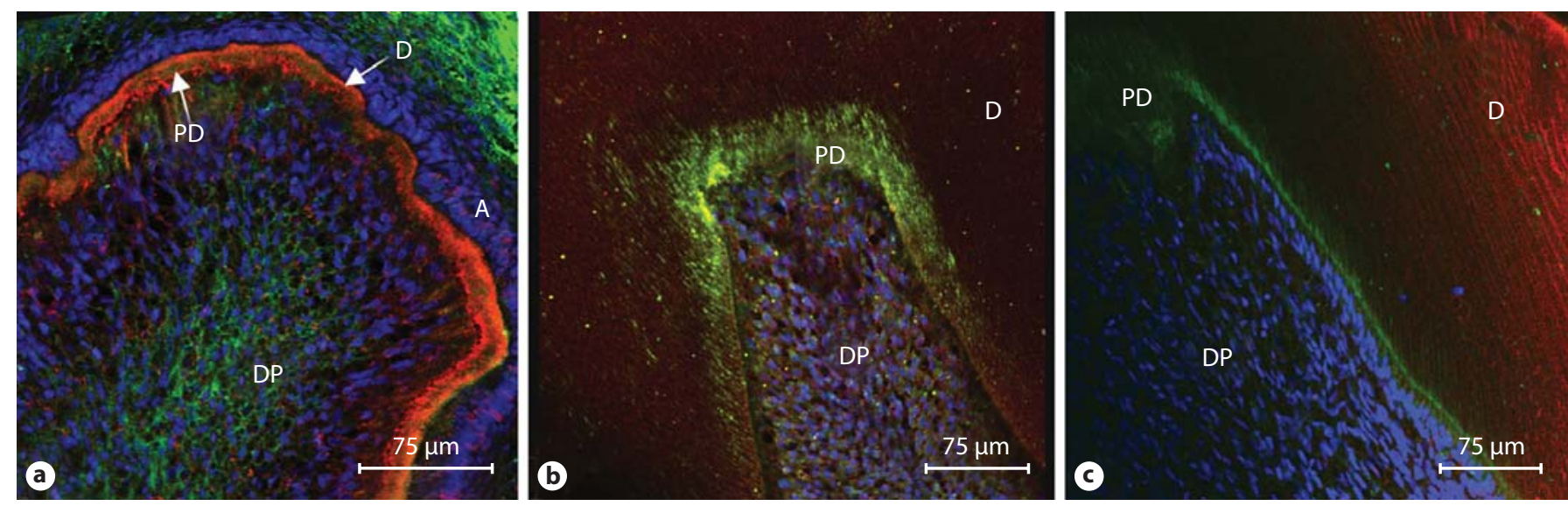

Fig. 1. IHC staining of DMP1 in the tooth. Sections were from the first mandibular molar of newborn (a), 3-week-old (b) and 8week-old rats (c). Alexa 488-labeled anti-rabbit $\mathrm{F}(\mathrm{ab})_{2}$ fragment (green) was used to recognize anti-DMP1-N-859 polyclonal antibody, while Alexa 546-labeled anti-mouse $\mathrm{F}(\mathrm{ab})_{2}$ fragment (red) was used to recognize anti-DMP1-C-clone 8G10.3 monoclonal antibody. The nuclei of cells were stained by TO-PRO-3 fluorescent dye (blue). The signal for the $\mathrm{NH}_{2}$-terminal fragment of $\mathrm{DMP} 1$ (green) was detected in the predentin (PD, arrow), whereas that for the $\mathrm{COOH}$-terminal fragment (red) was primarily observed in the mineralized dentin ( $\mathrm{D}$, arrow). $\mathrm{A}=$ Ameloblasts; $\mathrm{DP}=$ dental pulp.
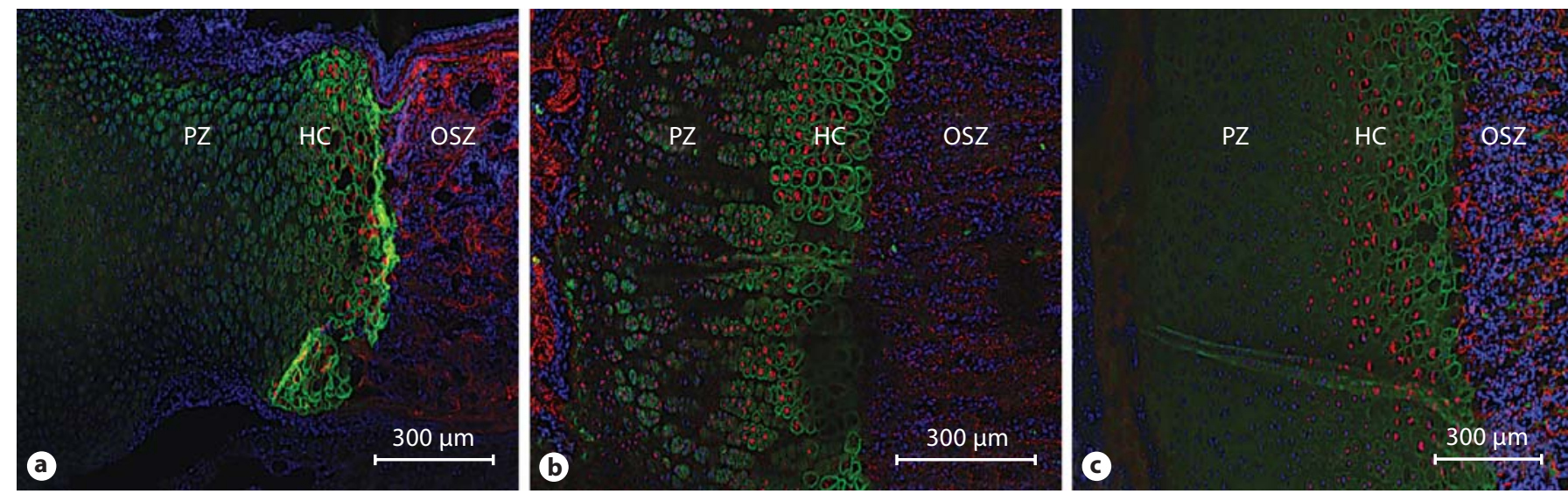

Fig. 2. IHC staining of DMP1 in the bone. Sections were from the epiphyseal regions of the humerus of newborn (a), 3-week-old (b) and 8 -week-old rats (c). The primary and secondary antibodies as well as nucleus-staining fluorophores were the same as in figure 1 . The $\mathrm{NH}_{2}$-terminal fragment (green) was primarily detected

\section{Results}

\section{DMP1 Fragments in the Tooth}

In the newborn rats, predentin was detected in the first molar along with a very thin layer of mineralized dentin. The $\mathrm{NH}_{2}$ - and $\mathrm{COOH}$-terminal fragments were observed in predentin (fig. 1a). Notably, the antibodies against the $\mathrm{COOH}$-terminal region of DMP1 strongly la- in the proliferation $(\mathrm{PZ})$ and prehypertrophic zones, while the $\mathrm{COOH}$-terminal fragment (red) accumulated mainly in the ossification zone (OSZ). Colocalization of the 2 fragments was observed in hypertrophic chondrocytes (HC). eloblasts overlapped with the nuclei. At the ages of 3 and 8 weeks, the differences in the localization of the 2 fragments of DMP1 became more distinct (fig. $1 b, c)$ : the $\mathrm{NH}_{2}$-terminal fragment of DMP1 was predominantly present in the predentin, while the $\mathrm{COOH}$-terminal fragment was present mainly in the mineralized dentin. Although the $\mathrm{COOH}$-terminal fragment of DMP1 was ob- 
served in the entire layer of mineralized dentin, the dentin in the peritubular areas stained more intense than those in the intertubular region.

\section{DMP1 Fragments in the Long Bone}

In the humerus, the differences in the localization of the $\mathrm{NH}_{2}$ - and $\mathrm{COOH}$-terminal fragments of DMP1 were already apparent in the newborn animals (fig. 2a): the $\mathrm{NH}_{2}$-terminal fragment was localized predominantly in the resting, proliferation and prehypertrophic zones of the metaphyseal growth plate and epiphyseal cartilage, while the $\mathrm{COOH}$-terminal fragment accumulated mainly in the calcification front and ossification zone. For all 3 age groups (newborn, 3 and 8 weeks), this specific, zone-dependent distribution pattern of the $\mathrm{NH}_{2}$ - and $\mathrm{COOH}$-terminal fragments of DMP1 remained the same (fig. 2). The colocalization of these 2 fragments was only observed in the hypertrophic chondrocytes.

\section{DMP1 Fragments in the Cellular Compartments of Osteocytes}

In osteocytes, DMP1 fragments showed difference in distribution between the cytoplasm and nuclei. $\mathrm{The} \mathrm{NH}_{2}-$ terminal fragment was observed along the cell membrane and the processes of osteocytes, while the $\mathrm{COOH}$-terminal fragment was concentrated in the central region of the cells, often overlapping with cell nuclei (fig. 3a). A trace amount of the full-length DMP1 (stained yellow) appeared in the cytosol in close proximity with the nuclei.

\section{DMP1 Fragments in the Cellular Compartments of HEK-293 and MC3T3-E1 Cells}

The IHC staining of nontransfected HEK-293 cells showed a negative reaction to both anti-DMP1-N-859 and anti-DMP1-C-8G10.3/anti-DMP1-C-857 antibodies (fig. 3b), while cells transfected with the DMP1 construct showed clear positive immunoreactions for both the $\mathrm{COOH}$-terminal (fig. 3c) and $\mathrm{NH}_{2}$-terminal fragment (fig. 3d). In the transfected HEK-293 cells, the COOHterminal fragment of DMP1 was localized in the nuclei, while the $\mathrm{NH}_{2}$-terminal fragment was distributed predominantly in the cytosol and accumulated around the nuclear envelope (fig. 3e).

In the nontransfected MC3T3-E1 cells, the COOHterminal fragment of DMP1 was primarily observed in the nuclei, while the $\mathrm{NH}_{2}$-terminal fragment was mainly found in the cytosol around the nucleus membrane (fig. 3f). The same distribution pattern of DMP1 fragments was observed in the MC3T3-E1 cells transfected with the DMP1 construct (fig. 3g); in the transfected cells, the full-length form of DMP1 (stained yellow) was more abundant than in the nontransfected cells.

It should be noted that in analyzing the distribution of DMP1 fragments in tissues and cells, consistent results were obtained by using different combinations of the antibodies: anti-DMP1-784, anti-DMP1-N-859, anti-DMP1785, anti-DMP1-C-clone 8G10.3 and anti-DMP1-C-857. Thus, we ruled out the possibility that the distribution differences in the DMP1 fragments might be due to nonspecific reactions of anti-DMP1 antibodies.

\section{Discussion}

The observations from this study indicate that the $\mathrm{NH}_{2}$ - and $\mathrm{COOH}$-terminal fragments derived from the full-length DMP1 are localized differently in tissues and cellular compartments.

In the tooth, the $\mathrm{NH}_{2}$-terminal fragment (likely DMP1-PG) accumulated in the nonmineralized predentin, while the $\mathrm{COOH}$-terminal fragment appeared more abundant in the mineralized dentin. Based on this observation, we hypothesize that after secretion, the phosphate-rich $\mathrm{COOH}$-terminal fragment is transported to the mineral nucleation sites of the organic framework where it mediates apatite crystal formation and growth. This speculation corroborates with previous reports that the COOH-terminal fragment of DMP1 promotes biomineralization [Tartaix et al., 2004; He et al., 2005; Gajjeraman et al., 2007]. The difference in localization between the $\mathrm{NH}_{2}$ - and $\mathrm{COOH}$-terminal fragments of DMP1 indicates that the $\mathrm{NH}_{2}$-terminal fragment (primarily in the form of DMP1-PG) may be progressively metabolized or removed before mineralization begins so that only the $\mathrm{COOH}$-terminal fragment remains in the mineralized dentin. This belief is in agreement with previous studies using $\left.{ }^{35} \mathrm{~S}\right]$ sulfate labeling, which demonstrated that nascent proteoglycans are lost when the predentin is converted to dentin [Prince et al., 1984; Steinfort et al., 1994].

In the humerus, we observed that the signal for the $\mathrm{NH}_{2}$-terminal fragment was primarily detected in the resting, proliferation and prehypertrophic zones, while that for the $\mathrm{COOH}$-terminal fragment was found in the calcification front and ossification zone. Although at present, we do not have sufficient data to explain these results, we speculate that both DMP1 fragments may be present in the nonmineralized cells of the cartilage, while the lack of $\mathrm{COOH}$-terminal fragment signal may be due 

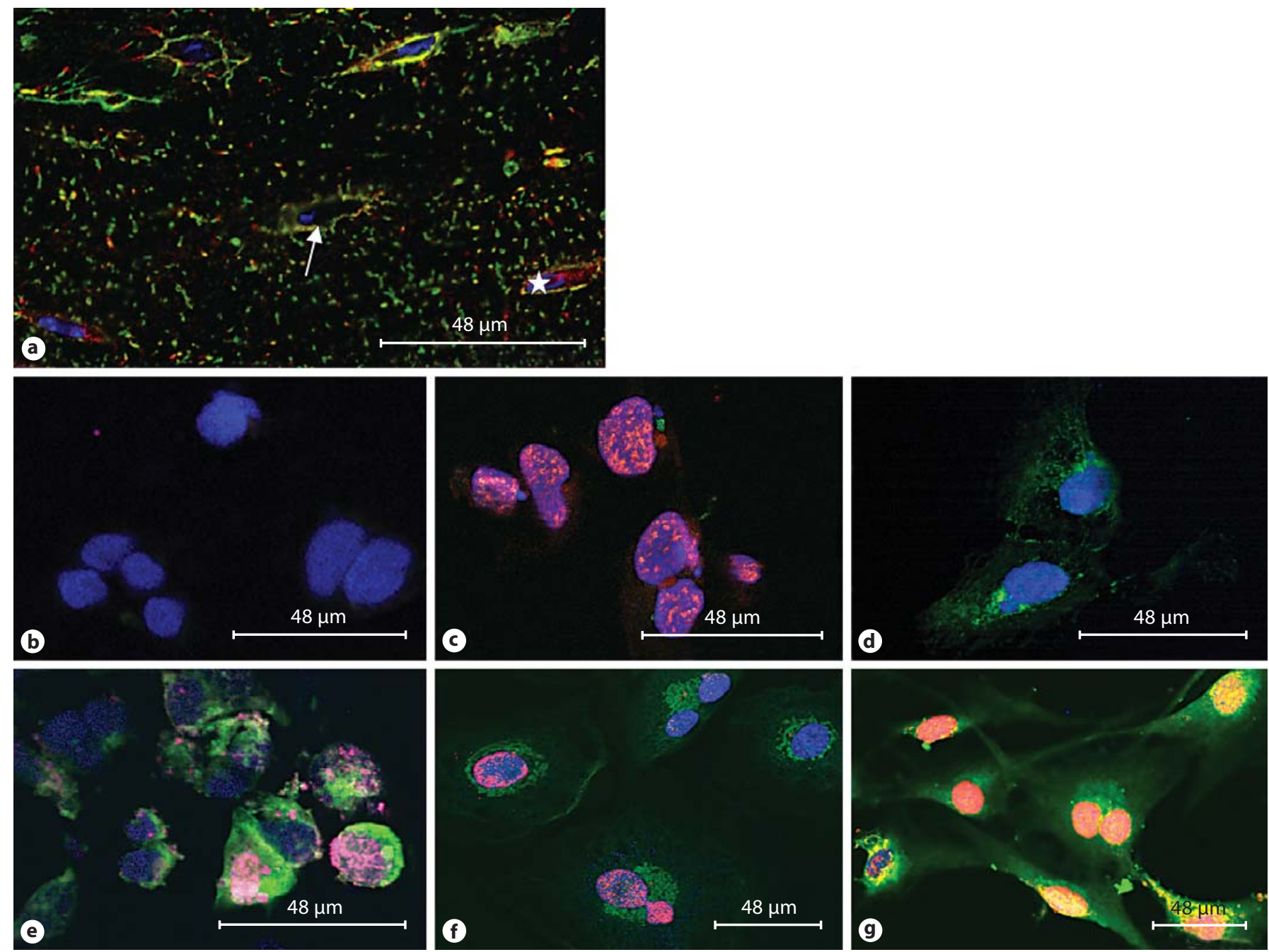

Fig. 3. IHC staining of DMP1 in osteocytes, HEK-293 and MC3T3E1 cells. Cell nuclei were stained by TO-PRO-3 fluorescent dye (blue). a DMP1 in osteocytes. The primary and secondary antibodies were the same as in figure 1 . The $\mathrm{NH}_{2}$-terminal fragment accumulated along the cell membrane and osteocyte processes (green, arrow), while the $\mathrm{COOH}$-terminal fragment (red, asterisk) was often seen overlapping with the nuclei (blue). b IHC staining of HEK-293 cells. Nontransfected cells were immunostained with anti-DMP1-C-clone 8G10.3 monoclonal antibody (positive reaction would give red fluorescence) and anti-DMP1N-859 polyclonal antibody (positive reaction would give green fluorescence). Note that only the nuclei showed positive fluorescent signal (TO-PRO-3, blue). c IHC staining of HEK-293 cells. Cells were transfected with DMP1 construct and immunostained with anti-DMP1-C-clone 8G10.3 monoclonal antibody (red). Note the presence of the COOH-terminal fragment of DMP1 in the nuclei (blue background). d IHC staining of HEK-293 cells. Cells were transfected with DMP1 construct and immunostained with anti-DMP1-N-859 polyclonal antibody (green). Note the presence of the $\mathrm{NH}_{2}$-terminal fragment of DMP1 in the cytosol around the nuclei. e IHC staining of HEK-293 cells (double staining). Cells were transfected with DMP1 construct and immunostained with anti-DMP1-C-clone 8G10.3 monoclonal antibody (red) and anti-DMP1-N-859 polyclonal antibody (green). Note the distribution difference between DMP1 fragments; also note their colocalization (yellow). $\mathbf{f}$ IHC staining of MC3T3-E1 cells (double staining). Nontransfected cells were immunostained with anti-DMP1-C-clone 8G10.3 monoclonal antibody (red) and antiDMP1-N-859 polyclonal antibody (green). Note the distribution difference between DMP1 fragments. g IHC staining of MC3T3E1 cells (double staining). Cells were transfected with DMP1 construct and immunostained with anti-DMP1-C-clone 8G10.3 monoclonal antibody (red) and anti-DMP1-N-859 polyclonal antibody (green). Note the distribution difference between DMP1 fragments. The intense yellow signal reflected the abundance of full-length DMP1, suggesting that these cells were unable to cleave the full-length DMP1 precursor quickly enough. 
to the fact that the epitopes of this fragment are hidden in the spatial structure of full-length DMP1. We further envision that the 'packaging' of the $\mathrm{COOH}$-terminal fragment inside the full-length form of DMP1 may serve a role in preventing these cells from being exposed to the highly acidic fragment. Clearly, further studies in this area are warranted to clarify these questions.

In the osteocytes, the $\mathrm{NH}_{2}$-terminal fragment of DMP1 accumulated in the cytosol and along the cell membrane and cell processes, whereas the $\mathrm{COOH}$-terminal fragment often overlapped with the cell nuclei. These findings suggest that the processed fragments of DMP1 may play different roles in transcriptional regulation and/or signaling. The nuclear localization of the nonphosphorylated full-length DMP1 has already been described by Narayanan et al. [2003]. In the present investigation, we observed an accentuated distribution of the $\mathrm{COOH}$-terminal fragment of DMP1 in the nuclei. Our observations suggest that the majority of the DMP1 molecules entering the nucleus are $\mathrm{COOH}$-terminal fragments. Sequence analysis of the $\mathrm{COOH}$-terminal fragment shows a lack of classical DNA-binding domains, making the speculation about its nuclear function more complicated. Based on the abundance of the $\mathrm{COOH}$-terminal fragment in the nucleus and the lack of a classical DNA-binding domain, it is tempting to believe that this acidic protein fragment may bind the basic histone complex in the nuclei.

\section{Acknowledgments}

This work was supported by NIH grant DE005092 (C.Q.). Dr. I. Maciejewska is on sabbatical at Baylor College of Dentistry from the Department of Oral Implantology, Medical University of Gdansk, Gdansk, Poland.

\section{References}

Baba, O., C. Qin, J.C. Brunn, J.N. Wygant, B.W. McIntyre, W.T. Butler (2004) Colocalization of dentin matrix protein 1 and dentin sialoprotein at late stages of rat molar development. Matrix Biol 23: 371-379.

Feng, J.Q., L.M. Ward, S. Liu, Y. Lu, Y. Xie, B. Yuan, X. Yu, F. Rauch, S.I. Davis, S. Zhang, H. Rios, M.K. Drezner, L.D. Quarles, L.F. Bonewald, K.E. White (2006) Loss of DMP1 causes rickets and osteomalacia and identifies a role for osteocytes in mineral metabolism. Nat Genet 38: 1310-1315.

Gajjeraman, S., K. Narayanan, J. Hao, C. Qin, A. George (2007) Matrix macromolecules in hard tissues control the nucleation and hierarchical assembly of hydroxyapatite. J Biol Chem 282: 1193-1204.

-George, A., B. Sabsay, P.A. Simonian, A. Veis (1993) Characterization of a novel dentin matrix acidic phosphoprotein: implications for induction of biomineralization. J Biol Chem 268: 12624-12630.

-He, G., S. Gajjeraman, D. Schultz, D. Cookson, C. Qin, W.T. Butler, J. Hao, A. George (2005) Spatially and temporally controlled biomineralization is facilitated by interaction between self-assembled dentin matrix protein 1 and calcium phosphate nuclei in solution. Biochemistry 44: 16140-16148.

Hirst, K.L., D. Simmons, J. Feng, H. Aplin, M.J. Dixon, M. MacDougall (1997) Elucidation of the sequence and the genomic organization of the human dentin matrix acidic phosphoprotein 1 (DMP1) gene: exclusion of the locus from a causative role in the pathogenesis of dentinogenesis imperfecta type II. Genomics 42: 38-45.

Distinct Compartmentalization of DMP1 Fragments
Lu, Y., L. Ye, S. Yu, S. Zhang, Y. Xie, M.D. McKee, Q Qin, C., B. Huang, J.N. Wygant, B.W. McIntyre, Y.C. Li, J. Kong, J.D. Eick, S.L. Dallas, J.Q. Feng (2007) Rescue of odontogenesis in Dmp1-deficient mice by targeted re-expression of DMP1 reveals roles for DMP1 in early odontogenesis and dentin apposition in vivo. Dev Biol 303: 191-201.

MacDougall, M., T.T. Gu, X. Luan, D. Simmons, J. Chen (1998) Identification of a novel isoform of mouse dentin matrix protein 1: spatial expression in mineralized tissues. J Bone Miner Res 13: 422-431.

Narayanan, K., R. Srinivas, A. Ramachandran, J. Hao, B. Quinn, A. George (2001) Differentiation of embryonic mesenchymal cells to odontoblast-like cells by overexpression of dentin matrix protein 1. Proc Natl Acad Sci USA 98: 4516-4521.

Narayanan, K., A. Ramachandran, J. Hao, G. He, K.W. Park, M. Cho, A. George (2003) Dual functional roles of dentin matrix protein 1 : implications in biomineralization and gene transcription by activation of intracellular $\mathrm{Ca}^{2+}$ store. J Biol Chem 278: 17500-17508.

-Prince, C.W., F. Rahemtulla, W.T. Butler (1984) Incorporation of $\left[{ }^{35} \mathrm{~S}\right]$ sulphate into glycosaminoglycans by mineralized tissues in vivo. Biochem J 224: 941-945.

Qin, C., J.C. Brunn, R.G. Cook, R.S. Orkiszewski, J.P. Malone, A. Veis, W.T. Butler (2003) Evidence for the proteolytic processing of dentin matrix protein 1: identification and characterization of processed fragments and cleavage sites. J Biol Chem 278: 3470034708 . C.H. McDonald, R.G. Cook, W.T. Butler (2006) A chondroitin sulfate chain attached to the bone dentin matrix protein $1 \mathrm{NH}_{2}$-terminal fragment. J Biol Chem 281: 80348040.

-Steinfort, J., R. van de Stadt, W. Beertsen (1994) Identification of new rat dentin proteoglycans utilizing C18 chromatography. J Biol Chem 269: 22397-22404.

Tartaix, P.H., M. Doulaverakis, A. George, L.W. Fisher, W.T. Butler, C. Qin, E. Salih, M. Tan, Y. Fujimoto, L. Spevak, A.L. Boskey (2004) In vitro effects of dentin matrix protein-1 on hydroxyapatite formation provide insights into in vivo functions. J Biol Chem 279: 18115-18120.

Ye, L., M. MacDougall, S.Zhang, Y.Xie, J.Zhang, Z. Li, Y. Lu, Y. Mishina, J.Q. Feng (2004) Deletion of dentin matrix protein-1 leads to a partial failure of maturation of predentin into dentin, hypomineralization, and expanded cavities of pulp and root canal during postnatal tooth development. J Biol Chem 279: 19141-19148.

Ye, L., Y. Mishina, D. Chen, H. Huang, S.L. Dallas, M.R. Dallas, P. Sivakumar, T. Kunieda, T.W. Tsutsui, A. Boskey, L.F. Bonewald, J.Q. Feng (2005) Dmp1-deficient mice display severe defects in cartilage formation responsible for a chondrodysplasia-like phenotype. J Biol Chem 280: 6197-6203. 